\title{
Electromagnetic Metamaterials to approach Superconductive-like Behaviour
}

\author{
A. Danisi ${ }^{\mathrm{a}, \mathrm{b}}$, C. Zannini ${ }^{\mathrm{a}}$ \\ ${ }^{\mathrm{a} C E R N}$, Geneva, Switzerland \\ ${ }^{b}$ currently at ITER, Saint-Paul-lez-Durance, France
}

\begin{abstract}
This paper underlines the possibility of using metamaterials to design an equivalent perfect electrical conductor. The design is formalized analytically using transmission line theory and supported by experimental measurements. This application of metamaterials could bring to the ground-breaking scenario of developing superconductive-like cavities and lossless guiding structures at ambient temperature.
\end{abstract}

\section{INTRODUCTION}

This paper addresses the possibility to nullify the surface impedance of a resistive wall by interposing a layer of metamaterial.

Strictly speaking, to nullify its impedance, the resistive wall has to behave as a perfect electric conductive material (PEC). This implies infinite equivalent conductivity and, therefore, zero equivalent surface impedance. A pipe with this special wall would behave as an ideal guiding structure, allowing wave propagation without losses. Likewise, a confined volume with the same special wall would behave as an ideal Radio-Frequency (RF) cavity. This behaviour is currently achieved by means of the use of superconductive materials. It is well known that these materials require cryogenics systems to keep their temperature below the critical limit value.

However, for many cases, as the two abovementioned examples, the nullification of the surface impedance would be required only in a narrow frequency bandwidth, to allow either wave propagation or resonance. This problem looks very well suited for metamaterials, since their properties are intrinsically narrow-band.

Section II describes the analytical treatment of the electromagnetic problem. The underlining equations can be used to properly design an equivalent loss-less structure in a certain frequency range.

Section III reports the experimental measurements already presented and analysed in [1]-[2] which supports the analytical findings.

Section IV outlines the potentially beneficial impact of this theory on currently relevant technologies where RF losses represent a significant design issue.

\section{THEORETICAL ANALYSIS}

\section{Transmission-line equivalence}

The theoretical analysis presented in this section reduces the geometry of the problem (depicted in Figure 1) to an equivalent transmission-line problem. As explained in [3], the equivalent wall impedance of such a geometry can be easily calculated by means of an impedance transportation of the wall surface impedance along the different equivalent transmission lines formed by the coating layers.

The condition of validity of the transmission line approach is that the equivalent surface impedance (computed transporting the layer impedances to the first interface) is constant with the incident wave direction. This condition is satisfied when the permittivity and permeability of the first layer are such that [4]

$$
\left|\varepsilon_{1} \mu_{1}\right| \gg \varepsilon_{0} \mu_{0}
$$

where $\varepsilon_{0}$ and $\mu_{0}$ denote vacuum permittivity and permeability. Similarly, $\varepsilon_{1}$ and $\mu_{1}$ are the complex permittivity and permeability of the interposed metamaterial layer (Figure 1). In this equivalence the transmission line can be considered closed on a surface impedance defined by

$$
\zeta_{S}=\frac{1+j}{\sigma \delta}
$$

where $\sigma$ and $\delta$ are the wall conductivity and penetration depth, respectively. In this framework, the penetration depth is defined as

$$
\delta=\sqrt{\frac{2}{\omega \sigma \mu_{2}}}
$$

where $\mu_{2}$ is the wall permeability, as per Figure 1, and $\omega$ is the angular frequency.

\section{Generic derivation of the transformed impedance}

Neglecting the cylindrical curvature of the structure in Figure 1, i.e. assuming high ratio between the cylinder

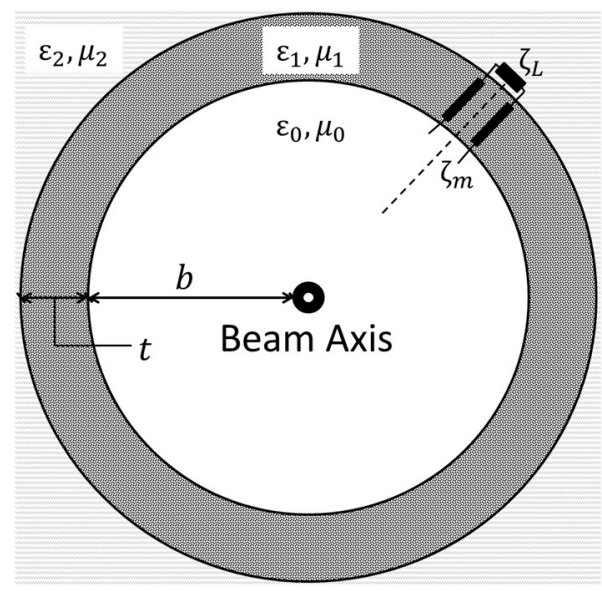

Figure 1: Transverse geometry of the problem. The metamaterial layer is put between the vacuum and the beam pipe (which extends to infinity). 
radius and the metamaterial layer thickness, the problem becomes a relatively simple condition of guided-wave propagation. The wave in the vacuum volume will "see" an equivalent impedance at the boundary with the metamaterial layer. This equivalent impedance corresponds to the surface impedance (previously defined), transformed along the equivalent transmission line of the metamaterial layer. In symbols,

$$
\zeta_{m}=\mathscr{N}\left(\zeta_{S}\right)
$$

where $\mathscr{T}$ denotes the impedance transformation function, operated on the load impedance (in this case, the surface impedance). In a transmission line, the impedance transformation along a certain line length $t$ can be formalised using the transmission line impedance formula [5], yielding for this case

$$
\zeta_{m}=\zeta_{r} \cdot \frac{\zeta_{S}+j \zeta_{r} \tan k_{r} t}{\zeta_{r}+j \zeta_{S} \tan k_{r} t}
$$

where $\zeta_{r}=\sqrt{\mu_{1} / \varepsilon_{1}}$ is the characteristic impedance of the metamaterial insertion, $k_{r}=\omega \sqrt{\mu_{1} \varepsilon_{1}}$ is the related propagation factor, $t$ is the layer thickness.

At this point, some observations need to be pointed out, for the treatment not to lack generality and, at the same time, to allow adequate simplification:

a) Given that the wall surface impedance is of the form given by eq. (2), the following notation can be adopted: $\zeta_{S}=a+j a$, with $a$ being a real and positive quantity; it is worth mentioning that this hypothesis is valid, for good conductors, until optical frequencies or above;

b) The characteristic impedance of the metamaterial insertion is in principle complex, i.e. $\zeta_{r}=b^{\prime}+j b^{\prime \prime}$; note that no assumption is made on the sign or magnitude of either real or imaginary part of this impedance;

c) As in any generic treatment of transmission line theory, the propagation factor is also considered complex; in particular, being always multiplied by the thickness in equation (5), the following notation can be adopted: $k_{r} t=\beta-j \alpha$, with $t$ being real and positive.

As a direct consequence of observation c), the tangent in equation (5) can be developed further, taking into account the following equivalence with hyperbolic functions [6]

$$
\tan (\beta-j \alpha)=\frac{\tan \beta-j \tanh \alpha}{1+j \tan \beta \tanh \alpha}
$$

Adopting the notations described above and the equivalence in (6), equation (5) can be written in expanded form and subsequently rationalised, to extract real and imaginary parts. For the sake of this paper, only the real part is the objective of the analytical treatment, since it corresponds to the resistive part of the transformed impedance, i.e. the impedance "seen" by the wave in the vacuum volume.

Before proceeding further, a brief justification is needed to whether the imaginary part is hereby not interesting.
Contrary to what happens to the real part, an imaginary load at the end of a transmission line can always be compensated with an element having equal and opposite reactance (e.g. a stub closed on a short, a lumped-element reactance). In addition, for the sake of this paper, the interest lies in investigating the possibility to nullify the real part, without really posing any condition on the imaginary part. In fact, considering a null real part, the load would be a purely imaginary impedance. As such, a purely imaginary load will always be characterized by a unitary reflection coefficient (in absolute value) with respect to a lossless line. It would exhibit a phase shift, which is however unimportant.

By virtue of the abovementioned observations, the real part of the transformed impedance is therefore analytically calculated as

$$
\begin{aligned}
\operatorname{Re}\left\{\zeta_{m}\right\}=\frac{\left(b^{\prime 2}+\right.}{} & \left.b^{\prime \prime 2}\right) \cdot N_{1} \\
D & \frac{\left(b^{\prime 2}-b^{\prime \prime 2}\right) \cdot N_{2}}{D} \\
& +\frac{\left(b^{\prime}+b^{\prime \prime}\right) \cdot N_{3}}{D} \\
& +\frac{\left(b^{\prime 3}-b^{\prime \prime 3}\right) \cdot N_{4}}{D} \\
& +\frac{2 a b^{\prime} b^{\prime \prime} \cdot N_{5}}{D}+\frac{2 a^{2} b^{\prime} \cdot N_{6}}{D} \\
& +\frac{2 b^{\prime} b^{\prime \prime}\left(b^{\prime}+b^{\prime \prime}\right) \cdot N_{7}}{D} \\
& -\frac{b^{\prime} b^{\prime \prime}\left(b^{\prime}-b^{\prime \prime}\right) \cdot N_{8}}{D} \\
& +\frac{2 a^{2} \cdot N_{9}}{D}
\end{aligned}
$$

where

$$
\begin{gathered}
D=\mid b^{\prime}-a(\tan \beta+\tanh \alpha) \\
+j[a(\tan \beta-\tanh \alpha) \\
\quad-\left(b^{\prime}+b^{\prime \prime}\right) \tan \beta \tanh \alpha \\
\left.+b^{\prime \prime}\right]\left.\right|^{2} \\
\quad-a \tan \beta \tanh \alpha \\
N_{1}=a-b^{\prime} \tanh \alpha-b^{\prime \prime} \tan \beta \\
N_{2}=a\left[(\tan \beta)^{2}+(\tanh \alpha)^{2}\right] \\
N_{3}=2 a b^{\prime}(\tan \beta)^{2}(\tanh \alpha)^{2} \\
N_{4}=-\tanh \alpha(\tan \beta)^{2} \\
N_{5}=(\tan \beta-\tanh \alpha)^{2} \\
N_{6}=\tan \beta(\tanh \alpha)^{2}-\tanh \alpha(\tan \beta)^{2} \\
N_{7}=\tan \beta(\tanh \alpha)^{2} \\
N_{8}=N_{4} \\
N_{9}=b^{\prime \prime} \tan \beta-b^{\prime} \tanh \alpha
\end{gathered}
$$

This analytical expression is quite general: it can be used for the extraction of the real part of a transformed impedance along any line length $t$, for any frequency, any interposing material properties (captured by $\mu_{1}$ and $\varepsilon_{1}$, which can be complex and/or negative signed), as long as observation a) is valid. This means that (7) can be used whenever it is needed to calculate the equivalent impedance of a metal when a coating layer of thickness $t$ is applied on top of it. 


\begin{tabular}{|c|c|}
\hline Parameter & Value \\
\hline Metal conductivity & $2 \cdot 10^{7} \mathrm{~S} / \mathrm{m}$ \\
\hline Metamaterial type & ENG (Negative electrical \\
permittivity)
\end{tabular}

Table 1: parameters for case study.

Before proceeding with the application of (7) to a metamaterial layer, a thorough benchmarking of the analytical formula has to be performed, in order to assess the consistency and coherence of (7) with respect to known scenarios. The known scenarios that shall be used are those which give rise to known results, i.e. the case of open and short loads, as well as the matched load case, all of them considered in a loss-less line.

For a short-circuit load in a loss-less line, $\alpha=0, a=$ $0, b^{\prime \prime}=0$, and therefore the coefficients given in (8) reduce to the following list

$$
\begin{aligned}
& D=\left|b^{\prime}\right|^{2} \\
& N_{1}=0 \\
& N_{2}=0 \\
& N_{3}=0 \\
& N_{4}=0 \\
& N_{5}=(\tan \beta)^{2} \\
& N_{6}=0 \\
& N_{7}=0 \\
& N_{8}=N_{4} \\
& N_{9}=0
\end{aligned}
$$

and therefore (7) becomes

$$
\operatorname{Re}\left\{\zeta_{m}\right\}=0
$$

which is in agreement with transmission line theory: the transport of a short-circuit load along a line always gives rise to a purely imaginary impedance.

Similar is the case of an open-circuit load in a loss-less line, whose analysis is skipped for the sake of conciseness $\left(\alpha=0, a \rightarrow \infty, b^{\prime \prime}=0\right)$.

The case of a matched load yields to $b^{\prime \prime}=b^{\prime}=a$. This means that the line has to be necessarily considered as a lossy line. In this case, analytical manipulations of (7) and (8), with due consideration of hypotheses a)-c) in Section II, bring to

$$
\operatorname{Re}\left\{\zeta_{m}\right\}=a
$$

which is consistent with transmission line theory for a matched line.

\section{Calculation of the transformed impedance for metamaterial layer}

Equation (7) gives the analytical basis on which the calculation of the transformation of a metal wall impedance along a metamaterial layer can be performed.

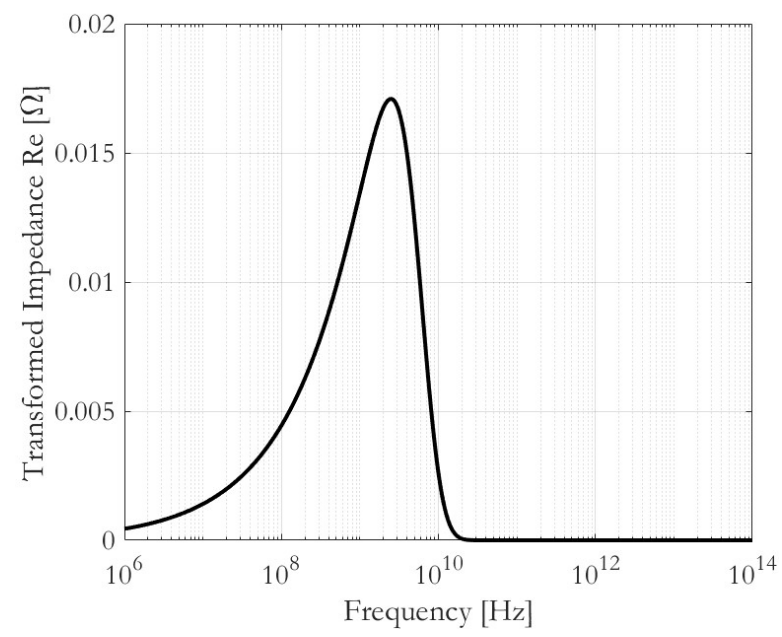

Figure 2: Real part of transformed impedance according to values in Table 1. Lossless case considered (i.e. real and negative value of electrical permittivity).

This allows to consider a practical example (illustrated in the values of Table 1) as a case study.

The values listed in Table 1 represent a rather generic example, where a good conductor (such as Titanium or Molybdenum) is "masked" with a 1-cm-thick layer of negative-permittivity (ENG) metamaterial. With these illustrative values, equation (7) can be used to predict and plot the real part of the transformed impedance. The results are depicted in Figure 2.

From these results, it is evident that above a certain frequency, the material starts to exhibit an equivalent behaviour of a Perfect Electrical Conductor (PEC), i.e. equivalent resistance approaching zero.

In fact, the usual dependence of the surface impedance with frequency is following the definition (2), which imposes an increasing trend. Instead, Figure 2 shows that, when approaching a certain frequency, this trend changes: the dependence defined in (2) is reversed by the metamaterial insertion, giving rise to a sort of transition. The frequency value around which this "metaconductive" transition happens, depends on material parameters, as well as engineering factors such as thickness and material manufacturing dimensions. However, it is clear from Figure 2 that the use of such metamaterials beyond the metaconductive transition would be beneficial whenever the needs to have extremely low resistive losses are important.

It is useful to notice that for frequencies reasonably beyond the metaconductive transition, Figure 2 shows a perfect nullification of the transformed resistance. This behaviour is maintained by virtue of the "lossless" hypothesis of the metamaterial insertion, i.e. the relative permittivity, even if negative, is purely real.

A more realistic model of an equivalent metamaterial would actually require to also take into account the imaginary part of the relative permittivity.

As to the value of such imaginary part, it is meaningful to consider reasonably small values, since the imaginary 


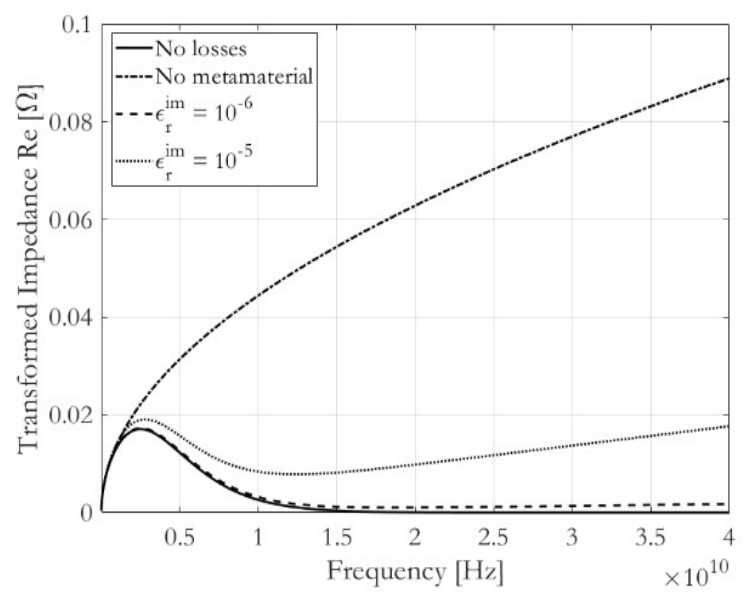

Figure 3: Real part of transformed impedance according to values in Table 1 for lossy cases.

part is normally associated to loss phenomena. The latter are commonly designed to be minimized, and therefore the imaginary part of the relative permittivity is hereby chosen to assume the values in Table 1, for comparison with the lossless case. These values correspond to very low loss metamaterials, yet considered to be fully synthesizable, e.g. [7].

Figure 3 shows the effect of finite imaginary relative permittivity on the metaconductive transition of the transformed real part of the impedance. It is useful to notice that, for this particular case, values of $10^{-5}$ are already enough to see a particularly relevant improvement (i.e. decrease) of the equivalent resistance, with respect to the "no-metamaterial" case. In fact, after a transition frequency of about $2 \mathrm{GHz}$, the equivalent resistance shows to be diminished by at least a factor 5 . With even lower losses, e.g. values of $10^{-6}$, the behaviour is found to even closely approach the lossless curve. In fact, in this case, about two orders of magnitude of difference can be observed between the equivalent resistance and the "nometamaterial" case.

\section{Simplified design equation for metaconductive surfaces}

Given the complete analytical treatment so far presented, it may be useful to provide a first-term design equation set to identify possible candidate materials for a certain application, or fix some engineering factors (e.g. thickness)

To this aim, it is convenient to write equation (7) in a simplified way, e.g. assuming no dielectric and magnetic losses. In these hypotheses, one can find that the transformed real part of the impedance is given by

$$
\operatorname{Re}\left\{\zeta_{m}\right\}=\zeta_{r} \cdot \frac{A\left[1+\left(\tan k_{r} t\right)^{2}\right]}{\left(A-\tan k_{r} t\right)^{2}+\left(\tan k_{r} t\right)^{2}}
$$

where

$$
A=\sigma \delta \zeta_{r}
$$

From the latter equations it can be seen that the equivalent behaviour of a PEC structure can be synthesized by simply imposing the following condition

$$
\tan \left(k_{r} t\right)=j
$$

This condition is fulfilled only if $k_{r} t$ is purely imaginary, which translates into the condition

$$
\varepsilon_{1} \cdot \mu_{1}<0
$$

This last condition can be satisfied exclusively with the use of a metamaterial, either of ENG or MNG type. In this latter case, equation (14) can be rewritten as

$$
\tanh \left(\left|k_{r} t\right|\right)=1
$$

This condition is exactly satisfied when $\left|k_{r} t\right| \stackrel{=}{\rightarrow} \infty$. However, the condition is practically reached for

$$
\left|k_{r} t\right| \geq 10
$$

It is worth reminding that, according to what has been found so far, $k_{r}$ is purely imaginary, and $\varepsilon_{1}, \mu_{1}$ are purely real, satisfying condition (15).

Equation (17) is the main design rule for metaconductive surfaces. Taking into account the definition of $k_{r}$ for a metamaterial satisfying (15), equation (17) can be rewritten as

$$
t \sqrt{\left|\varepsilon_{r 1}\right|} \geq \frac{10 c}{\omega_{0} \sqrt{\left|\mu_{r 1}\right|}}
$$

where $c=1 / \sqrt{\mu_{0} \varepsilon_{0}}$ is the speed of light in vacuum.

In this equation, $t$ is the needed thickness of the metamaterial layer with relative permittivity $\varepsilon_{r 1}$ and relative permeability $\mu_{r 1}$ to design a metaconductive surface for angular frequencies $\omega>\omega_{0}$.

Equation (18) identifies a design space for the metaconductive surfaces. For example, for ENG metamaterials $\left(\varepsilon_{r 1}<0\right.$ and $\left.\mu_{r 1}=1\right)$ this equation is corresponding to Figure 4 . The green zone represents the design space where the pairs $\left[t, \varepsilon_{r 1}\right]$ satisfy (18) and,

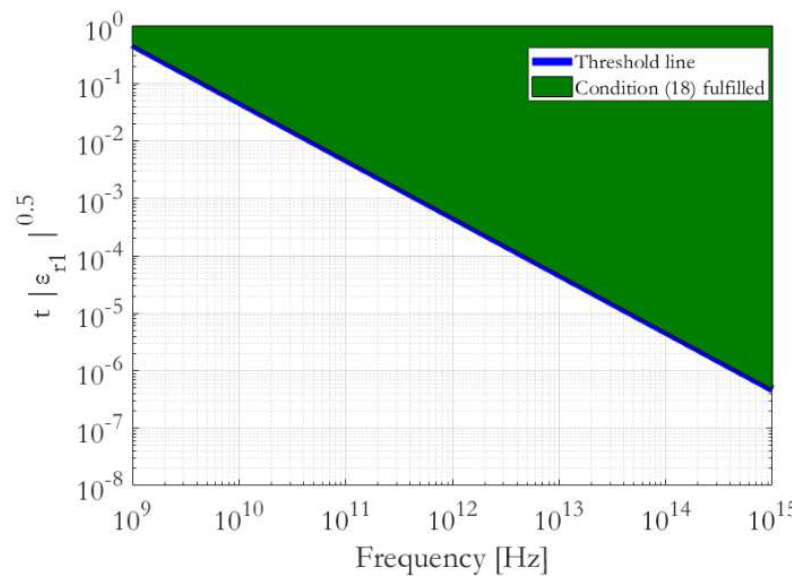

Figure 4: Design space for ENG metaconductive surfaces $\left(\varepsilon_{r 1}<0\right.$ and $\left.\mu_{r 1}=1\right)$ according to equation (18). Analogous design space can be plotted for MNG metamaterials $\left(\mu_{r 1}<0\right.$ and $\left.\varepsilon_{r 1}=1\right)$. 


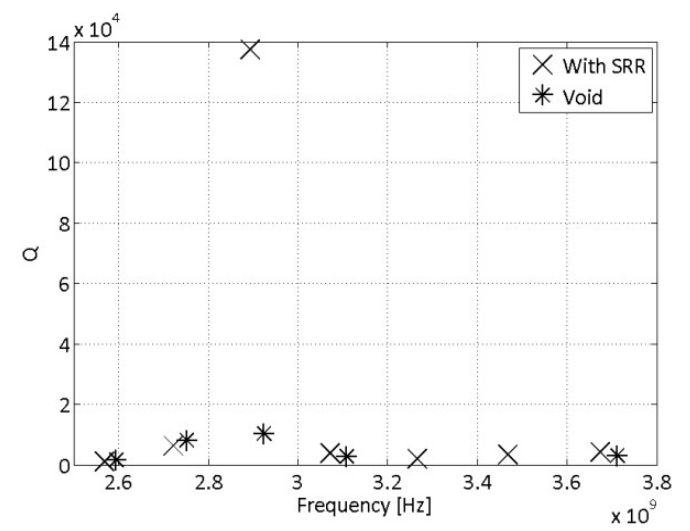

Figure 5: Measured unloaded quality factors (Q) with and without SRR insertions.

therefore, give rise to a metaconductive surface.

In reality, synthesized metamaterials exhibit negativepermittivity (ENG) or negative-permeability (MNG) behaviour in narrow frequency bandwidths [8]. Therefore, the metaconductive behaviour will be observed exclusively within these frequency bandwidths, provided that eq. (18) is satisfied.

To give a practical example of application of design equation (18), a potential metaconductive surface at a frequency of $\omega_{0}=2 \pi \cdot 48 \mathrm{GHz}$ would require a thickness $t=10 \mathrm{~mm}$ of an ENG metamaterial layer with $\varepsilon_{r 1}=-1$ and $\mu_{r 1}=1$ at the desired frequency.

Therefore, for those applications where the equivalent behaviour of a PEC (i.e. superconductive-like performance) is needed in a certain frequency range, equation (18) can be used for a preliminary selection and specification of the metaconductive surface. The complete exploration of the physical dependences is given by equation (7) instead.

\section{EXPERIMENTAL OBSERVATIONS}

The "metaconductive" behaviour, previously discussed analytically, could be experimentally verified measuring the surface impedance of a material structure as that of Figure 1.

A possible way to perform such a measurement with good resolution is to create a resonant empty cavity in the frequency range of interest, and subsequently measure the unloaded quality factor of the cavity modes. The unloaded quality factor of a resonance associated to an empty cavity is related solely to the losses on the conductive walls. Such losses are due to the surface impedance of the walls, which depends on the material conductivity and frequency, as previously analysed. The quality factor is inversely proportional to the surface resistance [9]. Therefore, for a metal cavity without any metamaterial insertion, the unloaded quality factor is directly related to the electrical conductivity of the metal.

Conversely, for a metal cavity with metamaterial insertion, the above-mentioned analytical treatment predicts that a significant decrease of the surface impedance should be observed in the frequency range where the metamaterial satisfies equation (18). This should result in a corresponding increase of the measured unloaded quality factor.

The measurement results and discussions detailed in the next sub-sections confirm the analytical findings.

\section{Measurements Results}

In the framework of investigating the use of metamaterial insertions for beam-coupling impedance mitigation, an experimental set-up with a waveguide has been built and presented in [2], together with the related measurements results.

The cavity has been obtained using a straight section of a rectangular copper waveguide WR284, enclosing it between two metallic plates and inserting a tiny antenna on one side, in order to excite the cavity modes.

Measurements have been performed with and without metamaterial insertions. The insertions are two split-ring resonators (SRRs) stripes on the cavity walls [2]. SRRs show negative relative permeability in a narrow bandwidth, around a well-defined resonance frequency [8]. Therefore, it is expected to observe an effect in this particular frequency bandwidth.

The results depicted in Figure 5 show that at about 2.9 $\mathrm{GHz}$ the measured unloaded quality factor is significantly higher in the case of metamaterial presence, meaning a decrease of an order of magnitude in the surface impedance. This important decrease of the surface impedance testifies the metaconductive behaviour of the measured cavity, in accordance with analytical findings.

The other peaks instead show little or no variation when the SRRs are put in the waveguide. This should not surprise since both material properties and the condition to get the equivalent behaviour of a perfect electrical conductor are frequency dependent (see eqs. (7) and (18)).

\section{CONCLUSIONS AND OUTLOOK}

The possibility of obtaining the equivalent behaviour of a perfect electrical conductive wall by means of metamaterial insertions has been analytically addressed. The required conditions to be satisfied in order to design the appropriate metamaterial insertions (e.g. thickness and material properties) are found analytically by means of a transmission-line model.

Existing experimental measurements conducted on a copper-wall Radio-Frequency cavity with metamaterial insertions have actually shown significant decline of the resistive losses. This represents an experimental evidence of the analytical predictions presented in this paper, i.e. that a properly-engineered metamaterial insertion could significantly diminish the resistive losses.

The authors consider that the idea proposed in this paper could be a first step towards the fascinating scenario of conceiving equivalent superconductive structures at room temperature, i.e. without the necessity to employ costly, complex and energy-consuming cryogenic systems. 


\section{REFERENCES}

[1] A. Danisi, C. Zannini, A. Masi, R. Losito and B. Salvant, "Theoretical analysis of metamaterial insertions for resistive-wall beam-coupling impedance reduction," proceedings of IPAC14, Dresden, Germany, June 2014.

[2] A. Danisi, C. Zannini, R. Losito, A. Masi, "Metamaterial insertions for resistive-wall beamcoupling impedance reduction," Accelerator Physics section, arXiv.org, Physics, arXiv:1910.02246. Available at https://arxiv.org/abs/1910.02246

[3] C. Zannini, "Electromagnetic simulations of CERN accelerator components and experimental applications", PhD thesis, Lausanne, EPFL, 2013. CERN-THESIS-2013-076.

[4] T. B. A. Senior and J. L. Volakis, Approximate boundary conditions in electromagnetics. 1995.

[5] D. M. Pozar, "Microwave Engineering," $4^{\text {th }}$ ed., Wiley, 2012, p. 272-316.

[6] I.N. Bronshtein, K.A. Semendyayev, Gerhard Musiol, Heiner Mühlig, "Handbook of Mathematics," Springer-Verlag Berlin Heidelberg, 2007.

[7] Wang P, Chen N, Tang C, et al. Engineering the Complex-Valued Constitutive Parameters of Metamaterials for Perfect Absorption. Nanoscale Res Lett. 2017;12(1):276. doi:10.1186/s11671-017-20482

[8] N. Engheta, R. W. Ziolkowski, "Metamaterials: Physics and Engineering Explorations", IEEE Press \& Wiley Interscience, 2006.

[9] E. L. Ginzton, "Microwave Measurements," Literary Licensing, 2012.

Alessandro Danisi was born in 1985 in Italy. He obtained the Ms. C. in Electronics Engineering in 2009 from University of Naples "Federico II" and the Ph. D. in Engineering in 2013 from the Swiss Federal Institute of Technology in Lausanne (EPFL). He has worked at CERN as Electromagnetics Engineer from 2008 to 2016, dealing with low-frequency magnetic sensors modelling and simulation, radio-frequency simulations for beamcoupling impedance problems, as well as research on metamaterials for accelerators. From 2016 to 2018, he was with the European Patent Office, working as examiner in the field of Applied Physics. From 2018, he is a staff member at the International Thermonuclear Experimental Reactor (ITER) in Southern France, working as Diagnostics Engineer.

The views and opinions expressed herein do not necessarily reflect those of the ITER Organization. The research exposed in this paper is the result of a personal independent work.

Carlo Zannini was born in 1982 in Italy. He obtained the Ms. C. in Electronics Engineering in 2008 from University of Naples "Federico II" and the Ph. D. in Physics in 2013 from the Swiss Federal Institute of Technology in Lausanne (EPFL). He has worked at CERN as Accelerator Physicist from 2008 to 2015, dealing with radio-frequency analysis, simulations and measurements for beam-coupling impedance problems and material characterization, as well as RF analysis of metamaterials for accelerators. From 2016 to 2018, he was with AVO-ADAM, a CERN spin-off for the development of proton-therapy linear accelerators, working as RF engineer. From 2018, he is a staff member at the European Organization for Nuclear Research (CERN), working as Accelerator Physicist. 